\title{
Decentralized Dynamic State Estimation in Power Systems using Unscented Transformation
}

\author{
Abhinav Kumar Singh, Student Member, IEEE, and Bikash C. Pal, Fellow, IEEE
}

\begin{abstract}
This paper proposes a decentralized algorithm for real-time estimation of the dynamic states of a power system. The scheme employs phasor measurement units (PMUs) for the measurement of local signals at each generation unit; and subsequent state estimation using unscented Kalman filtering (UKF). The novelty of the scheme is that the state estimation at one generation unit is independent from the estimation at other units, and therefore the transmission of remote signals to a central estimator is not required. This in turn reduces the complexity of each distributed estimator; and makes the estimation process highly efficient, accurate and easily implementable. The applicability of the proposed algorithm has been thoroughly demonstrated on a representative model.
\end{abstract}

Index Terms - dynamic state estimation, decentralized, phasor measurement units, unscented transformation, Kalman filter.

\section{NOMENCLATURE}

$\mathbf{0}_{\alpha \times \beta} \quad$ denotes a zero matrix of size $(\alpha \times \beta)$

$\chi, \chi^{-} \quad$ a sigma point and a predicted-state sigma point, respectively

$\gamma^{-} \quad$ a predicted-measurement sigma point

$\hat{\boldsymbol{v}}, \hat{\boldsymbol{w}} \quad$ the estimated means of $\boldsymbol{v}$ and $\boldsymbol{w}$, respectively

$\hat{\boldsymbol{X}}, \hat{\boldsymbol{X}}^{-} \quad$ the estimated means of $\boldsymbol{X}$ and $\boldsymbol{X}^{-}$, respectively

$\hat{\boldsymbol{x}}, \hat{\boldsymbol{y}}^{-} \quad$ the estimated means of $\boldsymbol{x}$ and $\boldsymbol{y}^{-}$, respectively

$\boldsymbol{g}, \overline{\boldsymbol{g}}$ discrete and continuous forms of system differential functions, resp.

$\boldsymbol{h}$ a column vector of the system algebraic functions

$K \quad$ the Kalman gain matrix

$\boldsymbol{P}_{X y}^{-} \quad$ the estimated cross-correlation between $\boldsymbol{X}^{-}$and $\boldsymbol{y}^{-}$

$\boldsymbol{P}_{v}, \boldsymbol{P}_{w}$ the estimated covariance matrices of $\boldsymbol{v}$ and $\boldsymbol{w}$, respectively

$\boldsymbol{P}_{X}^{-}, \boldsymbol{P}_{y}^{-}$the estimated covariance matrices of $\boldsymbol{X}^{-}$and $\boldsymbol{y}^{-}$, respectively

$\boldsymbol{P}_{x v} \quad$ the estimated cross-correlation between $\boldsymbol{x}$ and $\boldsymbol{v}$

$\boldsymbol{P}_{X}, \boldsymbol{P}_{x}$ the estimated covariance matrices of $\boldsymbol{X}$ and $\boldsymbol{x}$, respectively

$\boldsymbol{Q}, \boldsymbol{R}$ the constant covariance matrices of $\boldsymbol{v}$ and $\boldsymbol{w}$, respectively

$\boldsymbol{u}$ a column vector of the inputs to the system

$\boldsymbol{V} \quad$ a column vector of the bus voltages, $V_{l} e^{\theta_{l}}, l=1,2, \ldots, N$; in p.u.

$\boldsymbol{v}, \boldsymbol{w}$ the process noise and the measurement noise column vectors, resp.

$\boldsymbol{x}, \boldsymbol{y}$ column vectors of the states and the observed measurements, resp.

$\boldsymbol{X}, \boldsymbol{X}^{-} \quad$ the augmented-state and the predicted-state random variables, resp.

$\boldsymbol{y}^{-} \quad$ the predicted-measurement random variable

$\boldsymbol{Y}$ the bus admittance matrix in p.u.,

$\delta \quad$ the rotor angle in rad

$\lambda_{0} \quad$ the absolute bounding value of $\lambda_{y}$

$\lambda_{y} \quad$ the normalized innovation ratio for the measurement $y$

$\omega, \omega_{b} \quad$ the rotor-speed in p.u., \& its base-value in rad/s, resp.

$\phi, \phi_{y}, \phi_{w}$ stator current phase, its measured value and its noise, resp., in rad

$\Psi_{1 \mathrm{~d}}, \Psi_{2 \mathrm{q}}$ the subtransient emfs due to $d \& q$ axes damper coils, resp., in p.u.

$\sigma_{\eta} \quad$ the standard deviation of $\eta$, for $\eta=V_{w}, \theta_{w}, I_{w}, \phi_{w}$ and $f_{w}$

$\theta$ the stator voltage phase in $\mathrm{rad}$

$\theta_{y}, \theta_{w}$ the measured value of $\theta$, and the associated noise, resp., in rad

$A_{x}, B_{x}$ the AVR exciter saturation constants in p.u.

$D$ the rotor damping constant in p.u.

$E_{d}^{\prime} \quad$ the transient emf due to flux in $q$-axis damper coil in p.u.

$E_{q}^{\prime} \quad$ the transient emf due to field flux linkages in p.u.

$E_{f d} \quad$ the field excitation voltage in p.u.

$H$ the generator inertia constant in $\mathrm{s}$

Abhinav Kumar Singh (a.singh11@imperial.ac.uk) and Bikash C. Pal (b.pal@imperial.ac.uk) are with the Control and Power Group, Department of Electrical and Electronic Engineering, Imperial College London, SW7 2BT, London. This work was supported by EPSRC, U.K., under Grant $\mathrm{EP} / \mathrm{G} 066477 / 1$.
I the stator current magnitude in p.u.

$i \quad$ refers to the $i^{t h}$ generation unit or the $i^{t h}$ bus in the power system $I_{y}, I_{w}$ the measured stator current magnitude, and its noise, resp., in p.u. $I_{d}, I_{q} \quad$ the $d$-axis and $q$-axis components of the stator current, resp., in p.u. $j, k, l \quad$ refer to $\sqrt{-1}$, the $k^{t h}$ time sample and the $l^{\text {th }}$ sigma-point, resp. $k_{a}, k_{x}, k_{p}$ AVR-regulator gain, AVR-exciter gain, and PSS gain, resp., in p.u. $M, N$ the number of generation units and the number of buses, resp.

$m, n \quad$ the number of elements in $\boldsymbol{x}$ and $\boldsymbol{X}$, resp.

$P_{L}, Q_{L}$ the active and reactive components of a load, respectively, in p.u.

$P_{s \tau}, P_{s \tau}^{\prime}$ the $\tau^{t h}$ PSS state and algebraic quantity, resp., in p.u., for $\tau=1,2,3$

$R_{s} \quad$ the armature resistance in p.u.

$T$ denotes the matrix transpose

$t, T_{0} \quad$ the system time, and the system sampling period, resp., in $\mathrm{s}$

$T_{d 0}^{\prime \prime}, T_{q 0}^{\prime \prime}$ the $d$-axis and $q$-axis subtransient time constants, respectively, in s

$T_{d 0}^{\prime}, T_{q 0}^{\prime}$ the $d$-axis and $q$-axis transient time constants, respectively, in s

$T_{a}, T_{r}$ the AVR time constants of the regulator and the filter, resp., in $\mathrm{s}$

$T_{m}, T_{e}$ the mechanical and electrical torque inputs, respectively, in p.u.

$T_{w}, T_{x}$ time constants of the PSS-washout and the AVR-exciter, resp., in s

$T_{\tau 1}, T_{\tau 2}$ the PSS's $\tau^{t h}$ stage lead and lag time constants, resp. in s, $\tau=1,2$

$V, V_{a}$ the stator voltage magnitude, \& AVR regulator voltage, resp.,in p.u.

$V_{r}, V_{s}$ the PSS output voltage, and the AVR-filter voltage, resp., in $\mathrm{s}$

$V_{y}, V_{w}$ the measured value of $V$, and the associated noise, resp., in p.u.

$V_{\text {ref }}$ the AVR reference voltage in p.u.

$X_{d}^{\prime \prime}, X_{q}^{\prime \prime}$ the $d$-axis and $q$-axis subtransient reactances, respectively, in p.u.

$X_{d}^{\prime}, X_{q}^{\prime}$ the $d$-axis and $q$-axis transient reactances, respectively, in p.u.

$X_{d}, X_{q}$ the $d$-axis and $q$-axis synchronous reactances, respectively, in p.u.

$X_{l s} \quad$ the armature leakage reactance in p.u.

$\arg \{C\}$ denotes the angle of a complex number $C$, in $\mathrm{rad}$

$\operatorname{diag}\{\boldsymbol{A}\}$ denotes a diagonal matrix of the elements in set $\boldsymbol{A}$

\section{INTRODUCTION}

$\mathbf{T}$ HE power transmission infrastructure in the developed world faces two major challenges: reliability and sustainability. The new technology of generation from wind and solar are filling the gap created by the retirement of fossil fuel based synchronous generations. It is inevitable that the power flow pattern in the transmission system is not going to be as predictable as in the case with centralized synchronous generation. There has not been significant investments in transmission infrastructure for many reasons over the last few decades. This has led to a situation of stressed transmission system which is increasingly likely to fail to meet the growing demand. The technology of computation and control plays an important role to avert the system outage. However, the majority of control and system monitoring tools of the energy management systems (EMSs) are based on steady state system model, which cannot capture the dynamics of power system very well. This limitation is primarily due to the dependency of EMSs on slow update rates of the SCADA systems.

With the advent of phasor measurement unit (PMU) based wide-area measurement systems (WAMSs), new dynamic security assessment (DSA) techniques for the evaluation of system security are being developed which are more effective in capturing system dynamics than the traditional EMSs [1]-[4]. 
An accurate information of the dynamic states of the system, obtained through dynamic state estimation (DSE) [5]-[12], lies at the core of these techniques. Thus, real-time DSE, facilitated by high-level deployment of PMUs, has become absolutely essential in order to carry out online security assessments.

The past studies in DSE are mostly based on linear schemes [6]-[9]. These schemes involve linearization of the system's differential and algebraic equations (DAEs), followed by the calculation of Jacobian matrices. The linearization introduces approximation-errors, which may become significant over time, especially for a complex and high-order power system [11]. Moreover, the calculation of Jacobian matrices is computationally expensive, as it has to be done at every iteration of the algorithm.

The drawbacks of linear schemes have been addressed in more recent research papers which propose the application of unscented transformation to eliminate linearization and calculation of Jacobians [10]-[12]. In [10], an unscented Kalman filter (UKF) based algorithm has been proposed for the DSE of a synchronous machine connected to an infinite bus only. In multi-machine system, this limitation has been addressed in [11] by using a centralized UKF which needs remote signals from all the machines in the power system. This method has its own limitations that many of the signals required for estimation, such as the rotor speed and the state variables of the excitation system, are difficult to measure. Even if these signals are measured somehow, it is difficult to ensure their transmission to a central location at a high sampling rate. Unless these problems are dealt with, these methods may not be applied to a practical system.

A distributed estimation scheme has been proposed in this paper to address the aforementioned problems. The key advantages of the proposed scheme are as follows:

- The signals required for estimation (which are the generator voltage and current) are easy to measure using PMUs.

- Each distributed estimator has to estimate only the local states of the corresponding generation unit. Therefore the estimator is very fast and its speed remains independent of the size of the system, unlike a centralized scheme.

- Remote signals need not be transmitted; therefore the estimation process is not affected by network problems such as transmission delays and losses. Also, the signal sampling rates are not limited by the network bandwidth.

- The state estimation for one generation unit is completely independent from the estimation for other units. Thus, the errors in estimation remain isolated and are easier to pinpoint than in a centralized estimation scheme.

- The PMUs only need to be installed at each generation unit, and most power stations have installation of PMUs.

The rest of the paper is organized as follows. The problem statement and an overview of the proposed scheme are given in Section II. Section III presents the theory of unscented transformation while a description of power system modeling is given in Section IV. The decentralized filtering equations are derived in Section $\mathrm{V}$ and Section VI presents case study of a 68-bus test model. Section VII concludes the paper.

\section{Problem Statement And methodology in BRieF}

It is assumed that the power system is represented using a set of continuous-time non-linear DAEs, given by (1):

$$
\dot{\boldsymbol{x}}(t)=\overline{\boldsymbol{g}}[\boldsymbol{x}(t), \boldsymbol{u}(t), \boldsymbol{v}(t)] ; \boldsymbol{y}(t)=\boldsymbol{h}[\boldsymbol{x}(t), \boldsymbol{u}(t), \boldsymbol{v}(t)]+\boldsymbol{w}(t)
$$

After sampling (1) at a sampling period $T_{0}$, one gets:

$$
\begin{gathered}
\frac{\boldsymbol{x}\left(k T_{0}\right)-\boldsymbol{x}\left((k-1) T_{0}\right)}{T_{0}}=\overline{\boldsymbol{g}}\left[\boldsymbol{x}\left((k-1) T_{0}\right), \boldsymbol{u}\left((k-1) T_{0}\right), \boldsymbol{v}\left((k-1) T_{0}\right)\right] ; \\
\boldsymbol{y}\left(k T_{0}\right)=\boldsymbol{h}\left[\boldsymbol{x}\left(k T_{0}\right), \boldsymbol{u}\left(k T_{0}\right), \boldsymbol{v}\left(k T_{0}\right)\right]+\boldsymbol{w}\left(k T_{0}\right)
\end{gathered}
$$

Rewriting $k T_{0}$ as $k$ and $(k-1) T_{0}$ as $k-1$, 21 gets converted into the discrete form given by (3).

$$
\begin{gathered}
\boldsymbol{x}(k)=\boldsymbol{x}(k-1)+T_{0}\{\overline{\boldsymbol{g}}[\boldsymbol{x}(k-1), \boldsymbol{u}(k-1), \boldsymbol{v}(k-1)]\} \\
\Rightarrow \boldsymbol{x}(k)=\boldsymbol{g}[\boldsymbol{x}(k-1), \boldsymbol{u}(k-1), \boldsymbol{v}(k-1)] ; \\
\boldsymbol{y}(k)=\boldsymbol{h}[\boldsymbol{x}(k), \boldsymbol{u}(k), \boldsymbol{v}(k)]+\boldsymbol{w}(k)
\end{gathered}
$$

In state estimation the state $\boldsymbol{x}(k)$ is treated as a random variable with an estimated mean $\hat{\boldsymbol{x}}(k)$ and an estimated covariance $\boldsymbol{P}_{x}(k)$. It may be inferred from (3) that the measurement noise $\boldsymbol{w}(k)$ is assumed to be additive, while the process noise $\boldsymbol{v}(k)$ is assumed to be non-additive and the state and measurement are non-linearly related to $\boldsymbol{v}(k)$ (as explained in Section V). The covariance matrices for both the noises are assumed to be constant, and are denoted as $\boldsymbol{Q}$ for $\boldsymbol{v}(k)$ and $\boldsymbol{R}$ for $\boldsymbol{w}(k)$. If the process noise $\boldsymbol{v}(k)$ is also treated as a state then it may be augmented with $\boldsymbol{x}(k)$ to give an augmented state random variable $\boldsymbol{X}(k)=\left[\boldsymbol{x}(k)^{T}, \boldsymbol{v}(k)^{T}\right]^{T}$ with estimated mean $\hat{\boldsymbol{X}}(\boldsymbol{k})$ and estimated covariance $\boldsymbol{P}_{X}(k)$, and (3) reduces to:

$$
\boldsymbol{X}(k)=\boldsymbol{g}[\boldsymbol{X}(k-1), \boldsymbol{u}(k-1)] ; \boldsymbol{y}(k)=\boldsymbol{h}[\boldsymbol{X}(k), \boldsymbol{u}(k)]+\boldsymbol{w}(k)
$$

Problem statement: Find $\hat{\boldsymbol{X}}(k)$ and $\boldsymbol{P}_{X}(k)$, given $\hat{\boldsymbol{X}}(k-1)$, $\boldsymbol{P}_{X}(k-1), \boldsymbol{g}, \boldsymbol{h}, \boldsymbol{u}(k-1), \boldsymbol{u}(k), \boldsymbol{y}(k), \boldsymbol{Q}$ and $\boldsymbol{R}$, under the constraints that:

- the algorithm is decentralized, that is, the algorithm for one generation unit should work independently from the algorithms for other units; and

- only those measurements may be used which are easily measurable using PMUs, and are locally available.

Stating the problem in simpler terms, an iterative algorithm for finding the real-time estimates of the mean and covariance of the states needs to be devised, provided the system DAEs, the inputs, the local PMU measurements, and all the noise covariances are available. The algorithm should be such that the estimation process for each generation unit remains independent of the other.

Methodology: A block diagram of the system and the proposed decentralized methodology for finding a solution for the aforementioned problem statement is shown in Fig. 1. Each generation unit is equipped with a PMU responsible for measuring various phasors associated with that unit, specifically the voltage and current phasors. Power systems usually operate at a near constant system frequency of 50 or $60 \mathrm{~Hz}$, and thus all the measured signals from the system have a fundamental harmonic component which is equal to the system frequency. Assuming that other harmonics are present in relatively small quantities, when the measured signals are sampled at more 


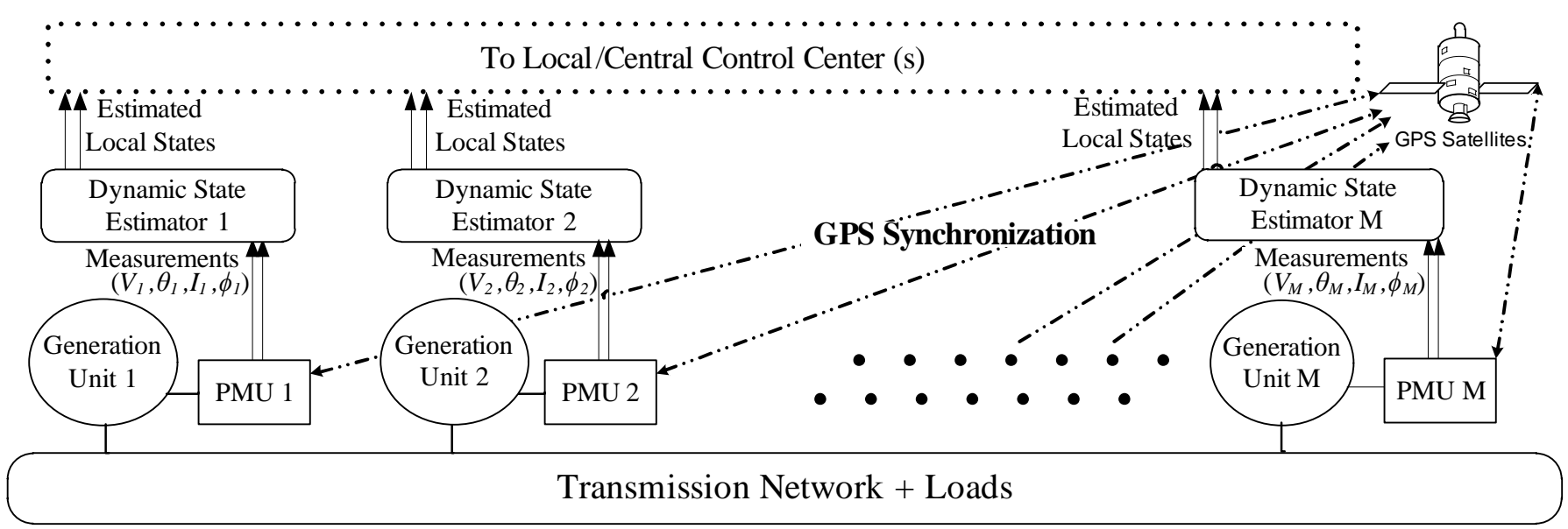

Fig. 1. System block-diagram and an overview of the methodology

than twice the system frequency, the sampling does not lead to any loss in information in the signals, as per the NyquistShannon sampling theorem. The PMUs provide sampling rates of over $600 \mathrm{~Hz}$ [13]; and hence they are capable of preserving the signal-information for state estimation purpose.

All of the PMUs in the power system are time synchronized to an absolute time reference provided by the global positioning system (GPS). The IEEE Standard for synchrophasor measurements for power systems specifies a basic time synchronization accuracy of $\pm 0.2 \mu$ s [14]. At 50 $\mathrm{Hz}$, this translates to a phase-measurement accuracy of around $\pm 0.06 \mathrm{mrad}$. Thus, the PMUs are expected to have an accuracy of around $\pm 0.1 \mathrm{mrad}$ for phase measurements.

The finite accuracy of a PMU for a given measurement is represented as a white-Gaussian noise superimposed over the correct value of the signal. Each noise is assumed to have a zero mean and a standard deviation equal to the accuracy of the PMU for the corresponding measurement. The sampled measurements, along with their noise variances, are sent from the PMU to the local estimator. The estimator is located in the vicinity of the PMU and hence the communication requirements are assumed to be easily met. The estimation is performed using the non-linear unscented transformation in conjugation with Kalman-like filtering. The obtained estimates of all the dynamic states of the machine are then sent to local and/or central control centers for taking control decisions.

\section{UNSCENTED TRANSFORMATION}

Unscented transformation was proposed by J. K. Uhlmann as a general method for approximating nonlinear transformations of probability distributions [15] . Based on the idea that it is easier to approximate a probability distribution than to approximate a non-linear function; this method is used to find consistent, efficient and unbiased estimates of the mean and covariance of a random variable undergoing a non-linear transformation [16]. If the non-linear transformation given by (4) is applied to $\boldsymbol{X}(k-1)$, then the estimated mean and covariance of the resultant state $\boldsymbol{X}(k)$ are derived in four steps:

1. Generation of sigma points: The first step is to generate a set of points, called as sigma points, whose sample mean and covariance are same as that of $\boldsymbol{X}(k-1)$. If the dimension of $\boldsymbol{X}(k-1)$ is $n$, then just $2 n$ sigma points, $\chi_{l}(k-1)$, $l=1,2, \ldots, 2 n$, need to be generated to capture its distribution [15]. The following algorithm is used for the generation of the sigma points [10]:

$$
\begin{gathered}
\chi_{l}(k-1)=\hat{\boldsymbol{X}}(k-1)+\left(\sqrt{n \boldsymbol{P}_{X}(k-1)}\right)_{l}, l=1,2, \ldots, n ; \\
\chi_{l}(k-1)=\hat{\boldsymbol{X}}(k-1)-\left(\sqrt{n \boldsymbol{P}_{X}(k-1)}\right)_{l}, l=(n+1),(n+2), \ldots, 2 n
\end{gathered}
$$

Here, $\left(\sqrt{n \boldsymbol{P}_{X}(k-1)}\right)_{l}$ is the $l^{\text {th }}$ column of the lower triangular matrix $\sqrt{n \boldsymbol{P}_{X}(k-1)}$, obtained by Cholesky decomposition, given by $n \boldsymbol{P}_{X}(k-1)=\sqrt{n \boldsymbol{P}_{X}(k-1)} \sqrt{n \boldsymbol{P}_{X}(k-1)}{ }^{T}$.

2. State prediction: In the second step the predicted-state sigma points are generated, which are given by $\chi_{l}^{-}(k)=$ $\boldsymbol{g}\left[\chi_{l}(k-1), \boldsymbol{u}(k-1)\right], l=1,2, \ldots, 2 n$. The sample mean and the sample covariance of these points are equal to the estimated mean, $\hat{\boldsymbol{X}}^{-}(k)$, and the estimated covariance, $\boldsymbol{P}_{X}^{-}(k)$, respectively, of a predicted-state random variable, $\boldsymbol{X}^{-}(k)$.

3. Measurement prediction: The third step is to generate the predicted-measurement sigma points, $\boldsymbol{\gamma}_{l}^{-}(k)=\boldsymbol{h}\left[\chi_{l}^{-}(k), \boldsymbol{u}(k)\right]$, $l=1,2, \ldots, 2 n$. The sample mean of these points is equal to $\hat{\boldsymbol{y}}^{-}(k)$, while the sum of $\boldsymbol{R}$ and the sample covariance of these points is equal to $\boldsymbol{P}_{y}^{-}(k)$. Here, $\hat{\boldsymbol{y}}^{-}(k)$ and $\boldsymbol{P}_{y}^{-}(k)$ are the estimated mean and the estimated covariance, respectively, of a predicted-measurement random variable, $\boldsymbol{y}^{-}(k)$. The crosscorrelation between the predicted-state sigma points and the predicted-measurement sigma points is equal to $\boldsymbol{P}_{X y}^{-}(k)$, the estimated cross-correlation between $\boldsymbol{X}^{-}(k)$ and $\boldsymbol{y}^{-}(k)$.

4. Kalman updation: The final step is to find $\hat{\boldsymbol{X}}(k)$ and $\boldsymbol{P}_{X}(k)$ using the normal Kalman filter equations [17]:

$$
\begin{gathered}
\boldsymbol{K}(k)=\boldsymbol{P}_{X y}^{-}(k)\left(\boldsymbol{P}_{y}^{-}(k)\right)^{-1} ; \hat{\boldsymbol{X}}(k)=\hat{\boldsymbol{X}}^{-}(k)+\boldsymbol{K}(k)\left(\boldsymbol{y}(k)-\hat{\boldsymbol{y}}^{-}(k)\right) \\
\boldsymbol{P}_{X}(k)=\boldsymbol{P}_{X}^{-}(k)-\boldsymbol{K}(k)\left[\boldsymbol{P}_{X y}^{-}(k)\right]^{T}
\end{gathered}
$$

The above four steps constitute the UKF. As stated in Section I the superiority of UKF has been established over other non-linear filters, such as the extended Kalman filter [18]. 


\section{Power System MODELING AND THE Discrete DAES}

The discrete DAEs of the power system derived using the continuous time DAEs given in [19], and a brief description of the various components of the system are as follows:

Generators: Each generator in the system has been represented using subtransient model [20]. The slow dynamics of the speed-governor have been ignored, treating the mechanical torque, $T_{m}$, as a constant parameter. The discrete DAEs for the $i^{\text {th }}$ generator are given by (18)-19] in the Appendix.

Excitation systems: Each generation unit may be excited manually or by using an automatic voltage regulator (AVR). Two types of AVRs have been considered in the case study. The discrete DAEs for the IEEE-DC1A type of AVR are given by (20), while for the IEEE-ST1A type of AVR they are given by (21). In the case of manual excitation, the field excitation voltage, $E_{f d}$, is equal to a constant reference, $V_{r e f}$.

Power system stabilizer (PSS): A PSS is used to provide supplementary damping control to the local modes of a generation unit. The transfer function of the PSS for the $i^{t h}$ generation unit, as considered in the case study, is given by $V_{s i}=k_{p}\left(\omega_{i}-1\right) \frac{\left(s T_{w i}\right)}{\left(1+s T_{w i}\right)} \frac{\left(1+s T_{11 i}\right)}{\left(1+s T_{12 i}\right)} \frac{\left(1+s T_{21 i}\right)}{\left(1+s T_{22 i}\right)}$. The discrete form of this transfer function is given by (22).

Network model: The network current balance equations for the generator buses are given by (23), while for the nongenerator buses the power balance equations are given by (24).

\section{PSEUdo INPUTS AND DECENTRALIZED FILTERS}

A generation unit consists of a generator, its AVR and PSS when present. The DAEs for a unit, given by (18)-(22), are coupled to the DAEs for other units through the network equations, given by (23)-24). The inputs to the power system come in the form of system-disturbances, such as load changes, linefaults and generation failures. If it is provided that none of the dynamic states are directly measured, a centralized state estimation scheme would require real-time information about all the system-wide disturbances, besides an information of the line parameters, the parameters for all the generation units and the system-wide PMU measurements. Obtaining such realtime information is practically not feasible. A decentralized scheme of estimation is the only practical alternative.

An inspection of (18)-(22) would reveal that the $i^{\text {th }}$ generation unit's $I, \phi$ and the dynamic states for the $(k+1)^{t h}$ sample are explicit functions of $V, \theta$ and the dynamic states for the $k^{t h}$ sample. This inspection leads to an idea which forms the basis of the decentralized estimation scheme: if $V$ and $\theta$ are treated as inputs, rather than as measurements, and $I$ and $\phi$ are treated as outputs (i.e. as normal measurements), then the dynamic equations for one generation unit can be decoupled from the dynamic equations for other units. It must be noted here that this representation is not unique, and the DAEs can be rearranged in such a way that $V$ and $\theta$ become the outputs, and $I$ and $\phi$ become the inputs. The idea is, therefore, to use one of the pair of measurements as the input pair, and the other pair as the output pair. In the rest of the paper, the pair of $V$ and $\theta$ is treated as the input pair.

The physical significance of the above idea may be understood by going deeper into the physics of power system dynamics. Any change or disturbance which takes place at one point in the power-system is reflected instantaneously throughout all the bus-voltages and currents. This is because the propagation of current and voltages is electromagnetic in nature, and hence is very fast. These voltage and current levels are in fact responsible for initiating the slower subtransient and transient dynamics of the devices which are connected to the buses. Therefore, just the knowledge of local bus-voltage and current is sufficient to predict and estimate the dynamics of the devices that are connected to that local bus; and in our case this device is a synchronous generator. But this knowledge of local voltage and current must be complete (both the magnitude and the phase are required), and this makes the synchronization of various PMU devices through the GPS satellites crucial to the estimation process. This synchronization of PMUs may also be considered as an indirect coordination between the decentralized estimators.

The idea of decoupling by treating $V$ and $\theta$ as inputs leads to a problem: only the measured values of $V$ and $\theta$ are available (given by $V_{y}$ and $\theta_{y}$, respectively), instead of their actual values, and hence they have associated noises, given by $V_{w}$ and $\theta_{w}$, , respectively. One way of including these noises in the DAEs is to model them as input noises [21]. But this would require linearization and would therefore defeat the purpose of unscented transformation and non-linear filtering. The other way of including the measurement noises is to redefine the values of $V$ and $\theta$ according to (7), based on the fact that the actual inputs are equal to the differences of their measured values and the associated noises.

$$
V_{i}(k)=V_{y i}(k)-V_{w i}(k) ; \theta_{i}(k)=\theta_{y i}(k)-\theta_{w i}(k) ;
$$

If the expressions for $V_{i}(k)$ and $\theta_{i}(k)$ from (7) are used in (18)-22), the resultant DAEs give the decentralized process model for the $i^{t h}$ generation unit, which is written in the following form, with $\boldsymbol{x}_{i}$ as the vector of the dynamic states, and $\boldsymbol{g}_{i}$ as the corresponding state functions:

$$
\boldsymbol{x}_{i}(k)=\boldsymbol{g}_{i}\left[\boldsymbol{x}_{i}(k-1), \boldsymbol{u}_{i}(k-1), \boldsymbol{v}_{i}(k-1)\right] ; i=1,2, \ldots, M
$$

In the above model, $\boldsymbol{u}_{i}(k-1)$ acts as a pseudo input vector and $\boldsymbol{v}_{i}(k-1)$ acts as a pseudo process noise vector, given as:

$$
\begin{gathered}
\boldsymbol{u}_{i}(k-1)=\left[V_{y i}(k-1), \theta_{y i}(k-1)\right]^{T} ; \\
\boldsymbol{v}_{i}(k-1)=\left[V_{w i}(k-1), \theta_{w i}(k-1)\right]^{T} ; i=1,2, \ldots, M
\end{gathered}
$$

$V_{w i}$ and $\theta_{w i}$ are white noises with zero means and constant standard deviations given by $\sigma_{V_{w i}}$ and $\sigma_{\theta_{w i}}$, respectively. Thus, the mean and covariance of $\boldsymbol{v}_{i}(k-1)$ also remain constant for each sample; and, for $i=1,2, \ldots, M$, they are given by:

$$
\hat{\boldsymbol{v}}_{i}(k-1)=\mathbf{0}_{2 \times 1} ; \boldsymbol{P}_{v i}(k-1)=\boldsymbol{Q}_{i}=\operatorname{diag}\left\{\sigma_{V_{w i}}{ }^{2}{ }{ }_{\theta_{w i}}{ }^{2}\right\}
$$

If $\hat{\boldsymbol{x}}_{i}(k-1)$ and $\boldsymbol{P}_{x i}(k-1)$ are the estimates of the mean and covariance of $\boldsymbol{x}_{i}(k-1)$; and $\boldsymbol{P}_{x v i}(k-1)$ is the cross-correlation between $\boldsymbol{x}_{i}(k-1)$ and $\boldsymbol{v}_{i}(k-1)$; and if $\boldsymbol{x}_{i}(k-1)$ is augmented with $\boldsymbol{v}_{i}(k-1)$ to give $\boldsymbol{X}_{i}(k-1)=\left[\boldsymbol{x}_{i}(k-1)^{T}, \boldsymbol{v}_{i}(k-1)^{T}\right]^{T}$, the estimates of the mean and covariance of $\boldsymbol{X}_{i}(k-1)$, for $i=$ $1,2, \ldots, M$, are given by:

$$
\hat{\boldsymbol{X}}_{i}(k-1)=\left[\begin{array}{l}
\hat{\boldsymbol{x}}_{i}(k-1) \\
\hat{\boldsymbol{v}}_{i}(k-1)
\end{array}\right] ;
$$




$$
\boldsymbol{P}_{X i}(k-1)=\left[\begin{array}{cc}
\boldsymbol{P}_{x i}(k-1) & \boldsymbol{P}_{x v i}(k-1)^{T} \\
\boldsymbol{P}_{x v i}(k-1) & \boldsymbol{P}_{v i}(k-1)
\end{array}\right]
$$

Rewriting (8) in the augmented state form, one gets:

$$
\boldsymbol{X}_{i}(k)=\boldsymbol{g}_{i}\left[\boldsymbol{X}_{i}(k-1), \boldsymbol{u}_{i}(k-1)\right] ; i=1,2, \ldots, M
$$

The equations for the measured magnitude, $I_{y i}$, and the measured phase, $\phi_{y i}$, of the stator current of the $i^{\text {th }}$ unit are:

$$
\begin{gathered}
I_{y i}(k)=\sqrt{\left(I_{q i}(k)\right)^{2}+\left(I_{d i}(k)\right)^{2}}+I_{w i}(k) ; \\
\phi_{y i}(k)=\arg \left\{I_{q i}(k)+j I_{d i}(k)\right\}+\delta_{i}(k)+\phi_{w i}(k) ; i=1,2, \ldots, M
\end{gathered}
$$

In (13), $I_{q i}(k)$ and $I_{d i}(k)$ are given by (19) after replacing the expressions of $V_{i}(k)$ and $\theta_{i}(k)$ from (77. Writing $\left[I_{y i}, \phi_{y i}\right]^{T}$ as the output vector $\boldsymbol{y}_{i}$, the corresponding measurement functions (given by (13), (7) and (19)) as $\boldsymbol{h}_{i}$, and $\left[I_{w i}, \phi_{w i}\right]^{T}$ as the output-noise vector $\boldsymbol{w}_{i}$, the measurement model comes out as:

$$
\boldsymbol{y}_{i}(k)=\boldsymbol{h}_{i}\left[\boldsymbol{X}_{i}(k), \boldsymbol{u}_{i}(k)\right]+\boldsymbol{w}_{i}(k) ; i=1,2, \ldots, M .
$$

The mean and covariance of $\boldsymbol{w}_{i}(k)$, for $i=1,2, \ldots, M$, are:

$$
\hat{\boldsymbol{w}}_{i}(k)=\mathbf{0}_{2 \times 1} ; \boldsymbol{P}_{w i}(k)=\boldsymbol{R}_{i}=\operatorname{diag}\left\{\sigma_{I_{w i}}{ }^{2}, \sigma_{\phi_{w i}}{ }^{2}\right\}
$$

The aggregate model for the $i^{t h}$ unit, given by (14) and (12), is the decentralized equivalent of (4). Also, the aggregate model for one unit is completely independent from other units. Thus, the four steps of UKF, as given in Section [III may be directly applied to the $i^{\text {th }}$ aggregate model to give its filtering algorithm, summarized as follows:

\section{Algorithm 1: Decentralized dynamic state estimation for the $i^{\text {th }}$ generation unit}

Begin Find $\boldsymbol{g}_{i}, \boldsymbol{h}_{i}, \boldsymbol{Q}_{i}$ and $\boldsymbol{R}_{i}$ according to 12, 14, 10) and 15, respectively. Let $m_{i}$ denote the total number of states to be estimated for the unit. Denote $n_{i}=m_{i}+2$. Denote the steady-state values of $\hat{\boldsymbol{x}}_{i}$ as $\boldsymbol{x}_{0 i}$.

While $(k \geq 1)$

\section{$\{$ STEP 1: Initialize}

if $(k==1)$ then initialize $\hat{\boldsymbol{x}}_{i}(0)=\boldsymbol{x}_{0 i}, \hat{\boldsymbol{v}}_{i}(0)=\mathbf{0}_{2 \times 1}, \boldsymbol{P}_{x i}(0)=\mathbf{0}_{m_{i} \times m_{i}}$, $\boldsymbol{P}_{x v i}(0)=\mathbf{0}_{2 \times m_{i}}, \boldsymbol{P}_{v i}(0)=\boldsymbol{Q}_{i}$ in 11 to get $\boldsymbol{P}_{X i}(0)$ and $\hat{\boldsymbol{X}}_{i}(0)$. else reinitialize $\hat{\boldsymbol{v}}_{i}(k-1)=\mathbf{0}_{2 \times 1}$ and $\boldsymbol{P}_{v i}(k-1)=\boldsymbol{Q}_{i}$, leaving rest of the elements in $\hat{\boldsymbol{X}}_{i}(k-1)$ and $\boldsymbol{P}_{X i}(k-1)$ unchanged.

STEP 2: Generate sigma points

$\chi_{i l}(k-1)=\hat{\boldsymbol{X}}_{i}(k-1)+\left(\sqrt{n_{i} \boldsymbol{P}_{X i}(k-1)}\right)_{l}, \quad l=1,2, \ldots, n_{i} ;$

$\chi_{i l}(k-1)=\hat{\boldsymbol{X}}_{i}(k-1)-\left(\sqrt{n_{i} \boldsymbol{P}_{X i}(k-1)}\right)_{l}, l=\left(n_{i}+1\right),\left(n_{i}+2\right), \ldots, 2 n_{i}$

STEP 3: Predict states

$\boldsymbol{\chi}_{i l}^{-}(k)=\boldsymbol{g}_{i}\left[\boldsymbol{\chi}_{i l}(k-1), \boldsymbol{u}_{i}(k-1)\right], l=1, \ldots, 2 n_{i} ; \hat{\boldsymbol{X}}_{i}^{-}(k)=\frac{1}{2 n_{i}} \sum_{l=1}^{2 n_{i}} \boldsymbol{\chi}_{i l}^{-}(k)$ $\boldsymbol{P}_{X i}^{-}(k)=\frac{1}{2 n_{i}} \sum_{l=1}^{2 n_{i}}\left[\boldsymbol{\chi}_{i l}^{-}(k)-\hat{\boldsymbol{X}}_{i}^{-}(k)\right]\left[\boldsymbol{\chi}_{i l}^{-}(k)-\hat{\boldsymbol{X}}_{i}^{-}(k)\right]^{T}$

STEP 4: Predict measurements

$\boldsymbol{\gamma}_{i l}^{-}(k)=\boldsymbol{h}_{i}\left[\boldsymbol{\chi}_{i l}^{-}(k), \boldsymbol{u}_{i}(k)\right], l=1,2, \ldots, 2 n_{i} ; \hat{\boldsymbol{y}}_{i}^{-}(k)=\frac{1}{2 n_{i}} \sum_{l=1}^{2 n_{i}} \boldsymbol{\gamma}_{i l}^{-}(k)$;

$\boldsymbol{P}_{y i}^{-}(k)=\frac{1}{2 n_{i}} \sum_{l=1}^{2 n_{i}}\left[\boldsymbol{\gamma}_{i l}^{-}(k)-\hat{\boldsymbol{y}}_{i}^{-}(k)\right]\left[\boldsymbol{\gamma}_{i l}^{-}(k)-\hat{\boldsymbol{y}}_{i}^{-}(k)\right]^{T}+\boldsymbol{R}_{i}$

$\boldsymbol{P}_{X y i}^{-}(k)=\frac{1}{2 n_{i}} \sum_{l=1}^{2 n_{i}}\left[\boldsymbol{\chi}_{i l}^{-}(k)-\hat{\boldsymbol{X}}_{i}^{-}(k)\right]\left[\boldsymbol{\gamma}_{i l}^{-}(k)-\hat{\boldsymbol{y}}_{i}^{-}(k)\right]^{T}$

STEP 5: Kalman update

$\boldsymbol{K}_{i}(k)=\boldsymbol{P}_{X y i}^{-}(k)\left(\boldsymbol{P}_{y i}^{-}(k)\right)^{-1} ; \hat{\boldsymbol{X}}_{i}(k)=\hat{\boldsymbol{X}}_{i}^{-}(k)+\boldsymbol{K}_{i}(k)\left(\boldsymbol{y}_{i}(k)-\hat{\boldsymbol{y}}_{i}^{-}(k)\right)$

$\boldsymbol{P}_{X i}(k)=\boldsymbol{P}_{X i}^{-}(k)-\boldsymbol{K}_{i}(k)\left[\boldsymbol{P}_{X i y}^{-}(k)\right]^{T}$

STEP 6: Output and time update

output $\hat{\boldsymbol{X}}_{i}(k)$ and $\boldsymbol{P}_{X i}(k)$

$k \leftarrow(k+1)$

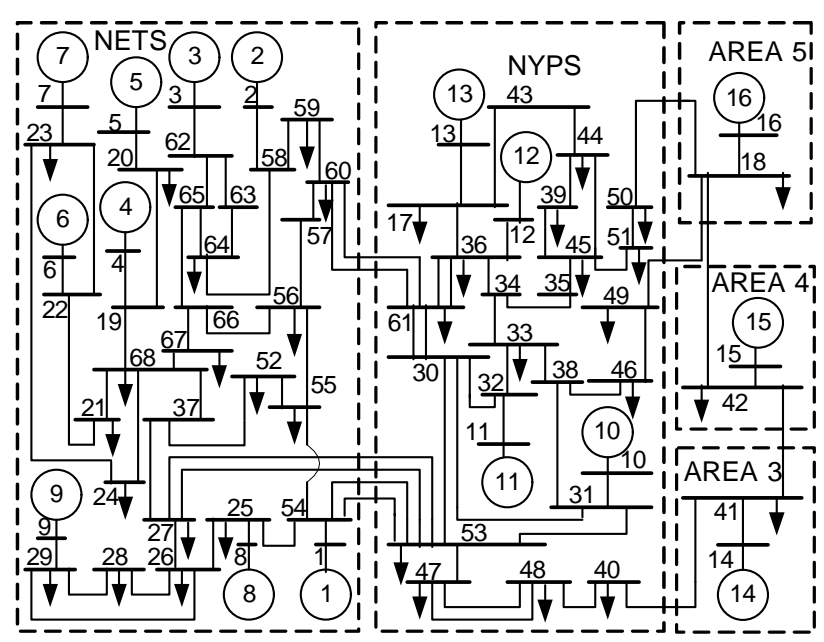

Fig. 2. Line diagram of the 16-machine, 68-bus, power system model

\section{CASE STUdy: 68 BUS TEST System}

A 16-machine, 68-bus test system, shown in Fig. 2 has been used for the case study. This system is a reduced order equivalent of the interconnected New England test system (NETS) and New York power system (NYPS) of 1970s. A detailed system description is available in [19], which is used to simulate the system in MATLAB on a personal computer with Intel Core 2 Duo, $2.0 \mathrm{GHz} \mathrm{CPU}$ and 2 GB RAM.

There are three types of generation units in the test system. The first eight units in the system are of type 1: with IEEEDC1A type of AVR, and without a PSS. The ninth unit is of type 2: with IEEE-ST1A type of AVR, and with a PSS installed. The rest of the units are of type 3: with manual excitation, and without a PSS. The state vectors for the $i^{t h}$ unit in the test system, according to these three types, are:

$\boldsymbol{x}_{i}=\left[\delta_{i}, \omega_{i}, E_{q i}^{\prime}, E_{d i}^{\prime}, \psi_{2 q i}, \psi_{1 d i}, V_{r i}, V_{a i}, E_{f d i}\right]^{T}, i=1,2, \ldots, 8$; $\boldsymbol{x}_{9}=\left[\delta_{9}, \omega_{9}, E_{q 9}^{\prime}, E_{d 9}^{\prime}, \psi_{2 q 9}, \psi_{1 d 9}, E_{f d 9}, P_{s 19}, P_{s 29}, P_{s 39}\right]^{T}$; and $\boldsymbol{x}_{i}=\left[\delta_{i}, \omega_{i}, E_{q i}^{\prime}, E_{d i}^{\prime}, \psi_{2 q i}, \psi_{1 d i}\right]^{T}, i=10,11, \ldots, 16$.

In the time-domain simulation, the actual values of $V, \theta, I$ and $\phi$ were sampled at $100 \mathrm{~Hz}\left(T_{0}=10 \mathrm{~ms}\right)$ and white Gaussian noises were added to them in order to generate measurements. Fig. 3 shows the generated $V_{y}$ and $\theta_{y}$ for the $13^{\text {th }}$ unit.

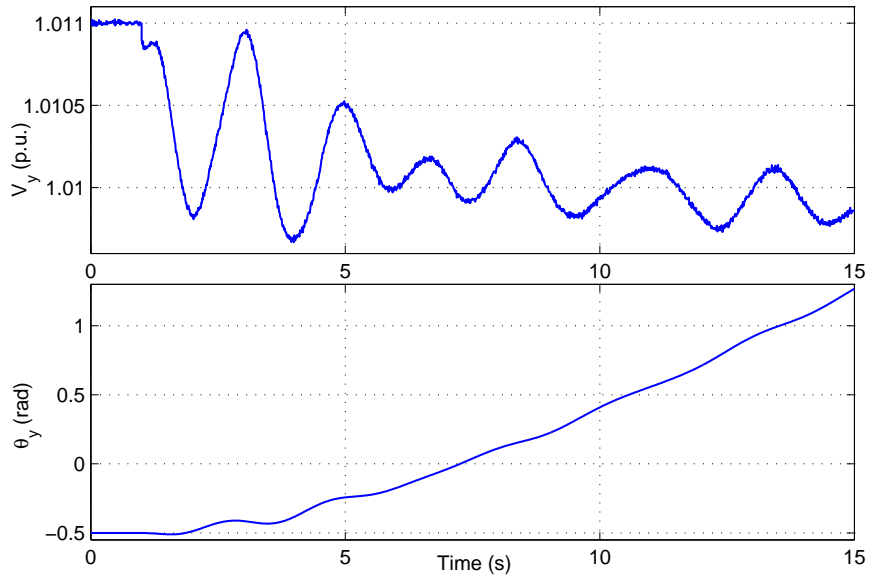

Fig. 3. Generated measurements for $V$ and $\theta$ for the $13^{\text {th }}$ generation unit 
As explained in Section III, the noises in the generated phase measurements were assumed to have standard deviation of $10^{-4} \mathrm{rad}$. Thus, $\sigma_{\theta_{w 0}}=\sigma_{\phi_{w 0}}=10^{-4} \mathrm{rad}$. Assuming one degree higher order of accuracy in measuring the manitudes $V$ and $I, \sigma_{V_{w 0}}=\sigma_{I_{w 0}}=10^{-5}$ p.u. The ' 0 ' in $\sigma_{V_{w 0}}, \sigma_{\theta_{w 0}}, \sigma_{I_{w 0}}$ and $\sigma_{\phi_{w 0}}$ denotes that these are base-case values. The variances for the generated noises for all the units were made equal to the base-case values, and hence $\boldsymbol{Q}_{i}=\boldsymbol{Q}_{0}=\operatorname{diag}\left\{10^{-10}, 10^{-8}\right\}$ and $\boldsymbol{R}_{i}=\boldsymbol{R}_{0}=\operatorname{diag}\left\{10^{-10}, 10^{-8}\right\} ; i=1,2, \ldots, 16$, from (10), 15.
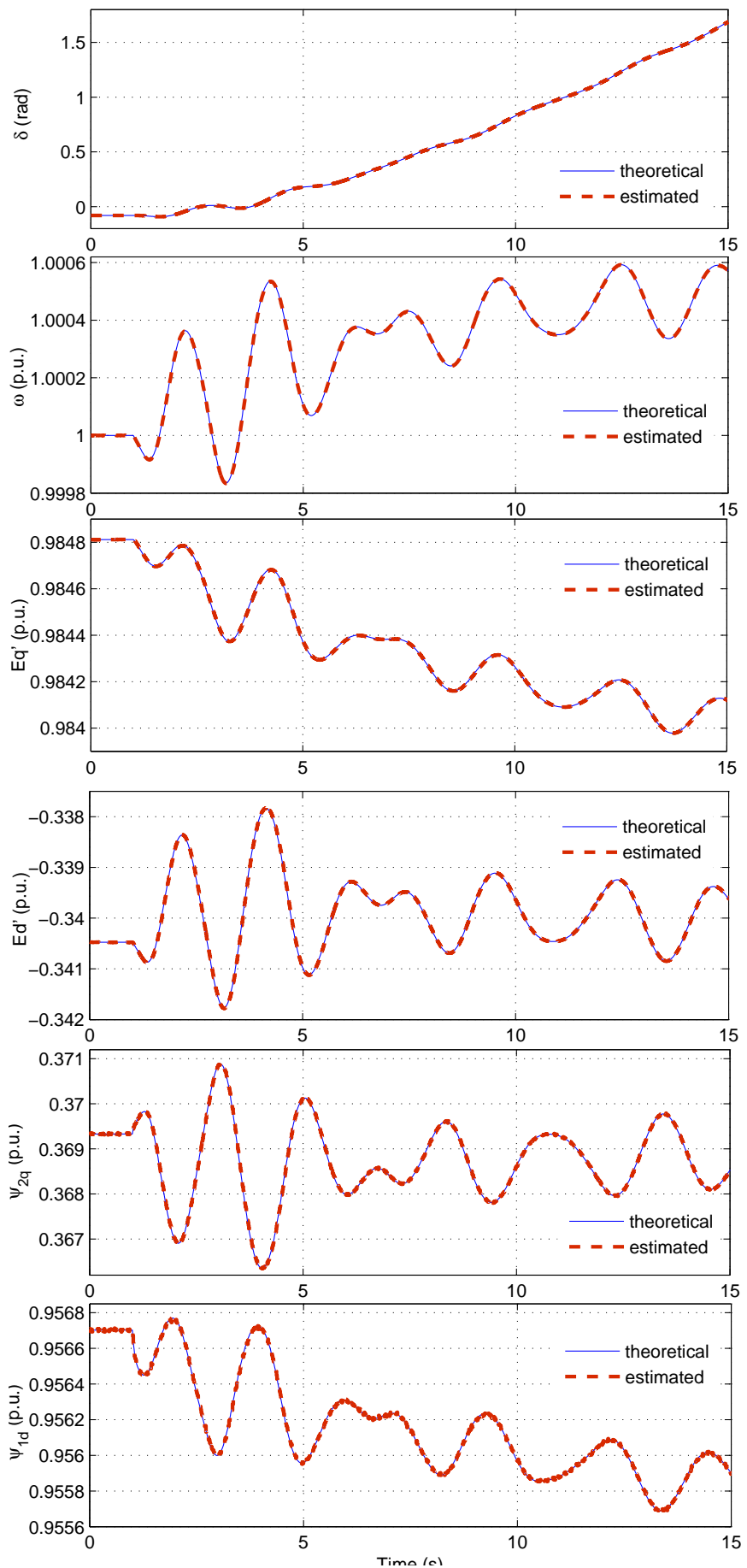

Fig. 4. Estimated vs theoretical values for dynamic states of the $13^{\text {th }}$ unit

In the starting of the simulation, the system was operating in a steady condition. Then at $t=1 \mathrm{~s}$, a disturbance was created by a three-phase fault and immediate outage of one of the tie-lines between buses 53-54. The $i^{t h}$ decentralized UKF algorithm, as given in Section $\mathrm{V}$ was running alongwith the simulation of the $i^{t h}$ unit. The generated measurements from each unit were given as input to the corresponding UKF. The theoretical states, alongwith their real-time estimated values, were plotted for each unit. Due to space-constraints, plots for only three units (of different types) have been shown: unit 13 of type 3 (Fig. 4), unit 9 of type 2 (Fig. 5), and unit 3 of type 1 (Fig. 6).

Estimation accuracy: It can be seen in Fig. 4 , 5 and 6 that for every dynamic state, the plot of estimated values almost coincides with those of the theoretical values. Thus, it is evident that the decentralized UKF scheme generates accurate estimates of all the dynamic states of a generating unit. As all the generator states have been estimated with high accuracies, they can be reliably used for further control and security decisions.

Sensitivity to noise: The noise variances assumed in the base-case are very conservative, and in real-life situations the measurement noises may have higher variances. Thus, the robustness of the proposed algorithm to higher noise variances was tested. For this, the variances $\boldsymbol{Q}_{i}$ and $\boldsymbol{R}_{i}$ were varied in multiples of tens of their base-case values, $\boldsymbol{Q}_{0}$ and $\boldsymbol{R}_{0}$, and the effect on estimation-accuracy was observed. Fig. 7 shows the effect of variations in noise-variances on the estimation of $\omega$ for the type 2 of generation unit. The plots have been shown for a portion of the total simulation time for the clarity. It is evident from Fig. 7 that the algorithm is robust,
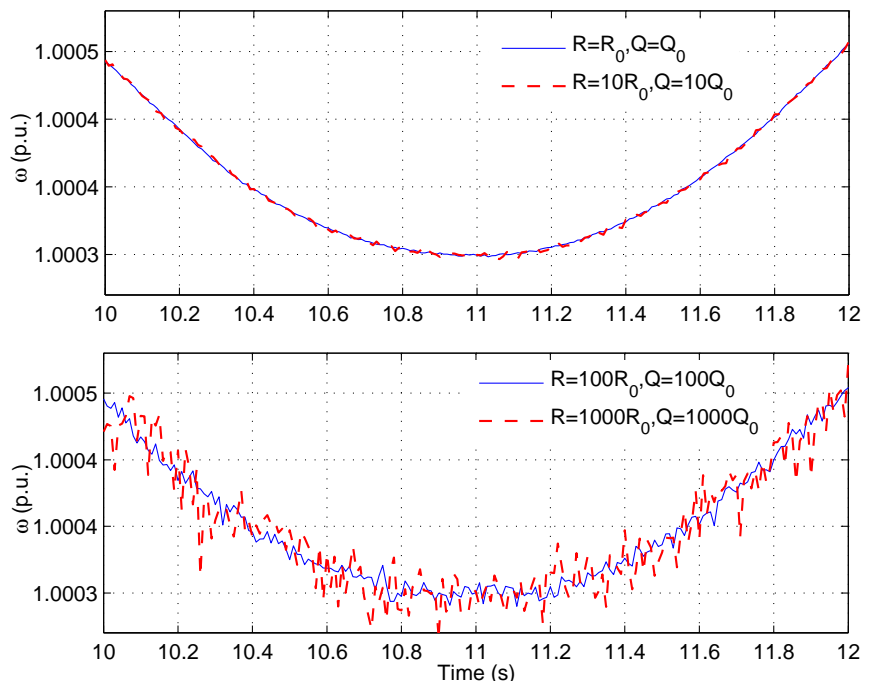

Fig. 7. Effect of noise variances on the accuracy of estimation

with minor errors in estimated values, even when the noisevariances are hundred times their base-case values. When the noise-variances are thousand times the base-case variances, the estimated states have significant errors and deviations, and hence become unusable.

Computational feasibility: The proposed algorithm was tested on two more standard IEEE test systems in order to assess its scalability. As the measurements are updated every $10 \mathrm{~ms}\left(T_{0}=10 \mathrm{~ms}\right)$, a single iteration of the algorithm should 
not require more than $10 \mathrm{~ms}$, otherwise the algorithm would not run in real-time. The average time for one iteration has been tabulated in Table $\prod$ for the three test systems. A centralized scheme for DSE (given in [11]) was also implemented on all the test systems, and the corresponding average iteration times have been tabulated. It can be inferred from Table I that the computational speed of the proposed decentralized algorithm is very fast and it remains independent of the size of the system, while the centralized algorithm becomes slow and infeasible for large systems (68-bus and 145-bus systems).

\section{BAD-DATA DETECTION}

PMU signals not only suffer from noise, but they are also prone to gross errors; and therefore a bad-data detection algorithm is required for the proposed decentralized estimator. Bad data detection in UKF is based on the fact that the ratio between the deviation of actual measurement from the
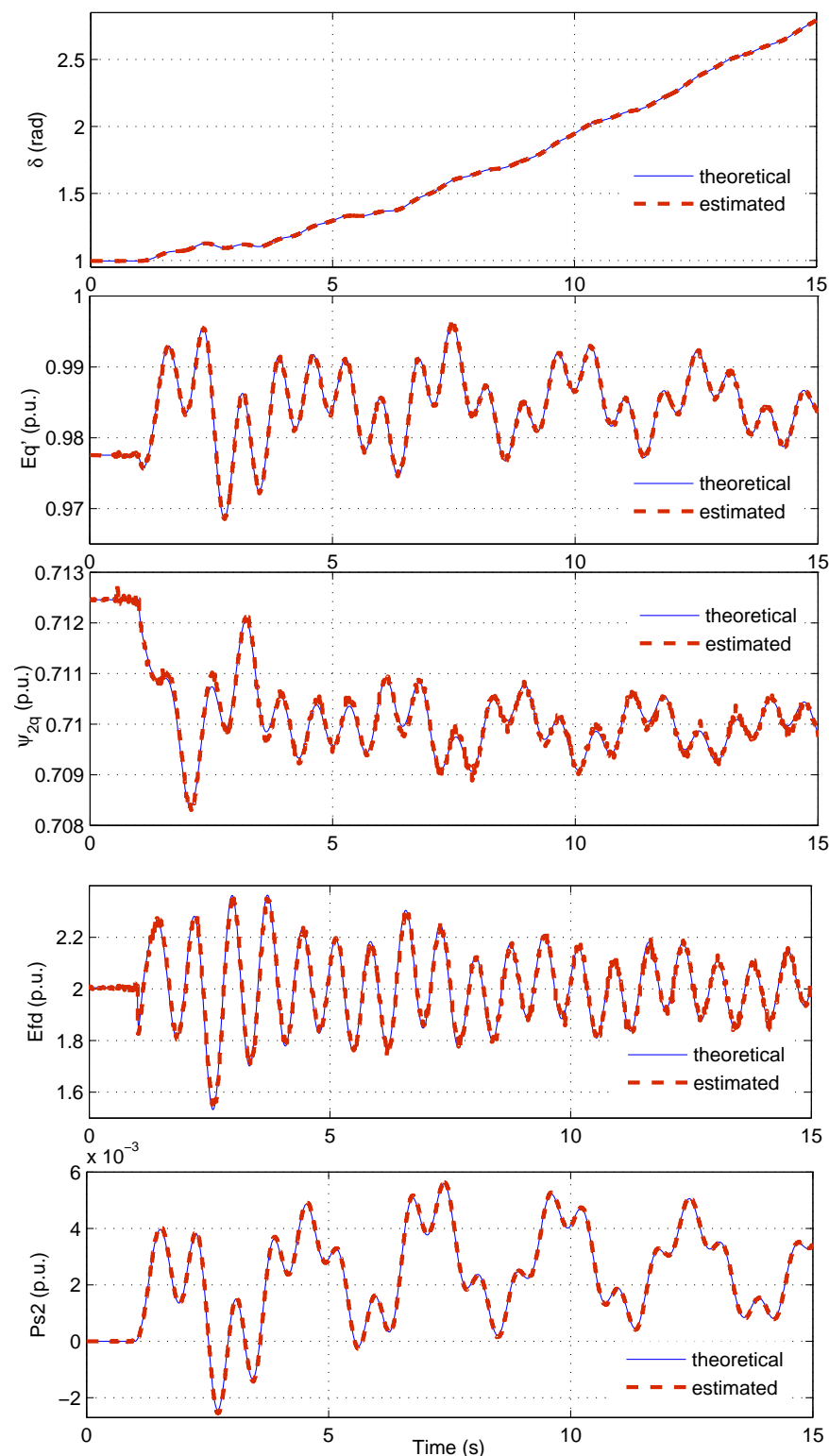

TABLE I

COMPARISON OF COMPUTATIONAL SPEED

\begin{tabular}{|l|c|c|}
\hline \hline & \multicolumn{2}{|c|}{ Average computational time for one iteration (in ms) } \\
\hline Test system & Decentralized algorithm & Centralized algorithm \\
\hline IEEE 30-bus & 0.33 & 1.45 \\
IEEE 68-bus & 0.33 & 12.4 \\
IEEE 145-bus & 0.33 & 139 \\
\hline
\end{tabular}

predicted measurement and the expected standard deviation of the predicted measurement remains bounded in a narrow band in the absence of any bad data; and this ratio is called as normalized innovation ratio [11], [12]. Mathematically, this fact may be stated using (16) and (17), where $\lambda_{y_{i, 1}}$ and $\lambda_{y_{i, 2}}$ are the normalized innovation ratios for the two measurements $y_{i, 1}=I_{y i}$ and $y_{i, 2}=\phi_{y i}$, respectively (Recall that $\left.\boldsymbol{y}_{i}=\left[I_{y i}, \phi_{y i}\right]^{T}\right) ; \hat{\boldsymbol{y}}_{i}^{-}=\left[\hat{y}_{i, 1}^{-}, \hat{y}_{i, 2}^{-}\right]^{T} ; P_{y i, 1}^{-}$is the first
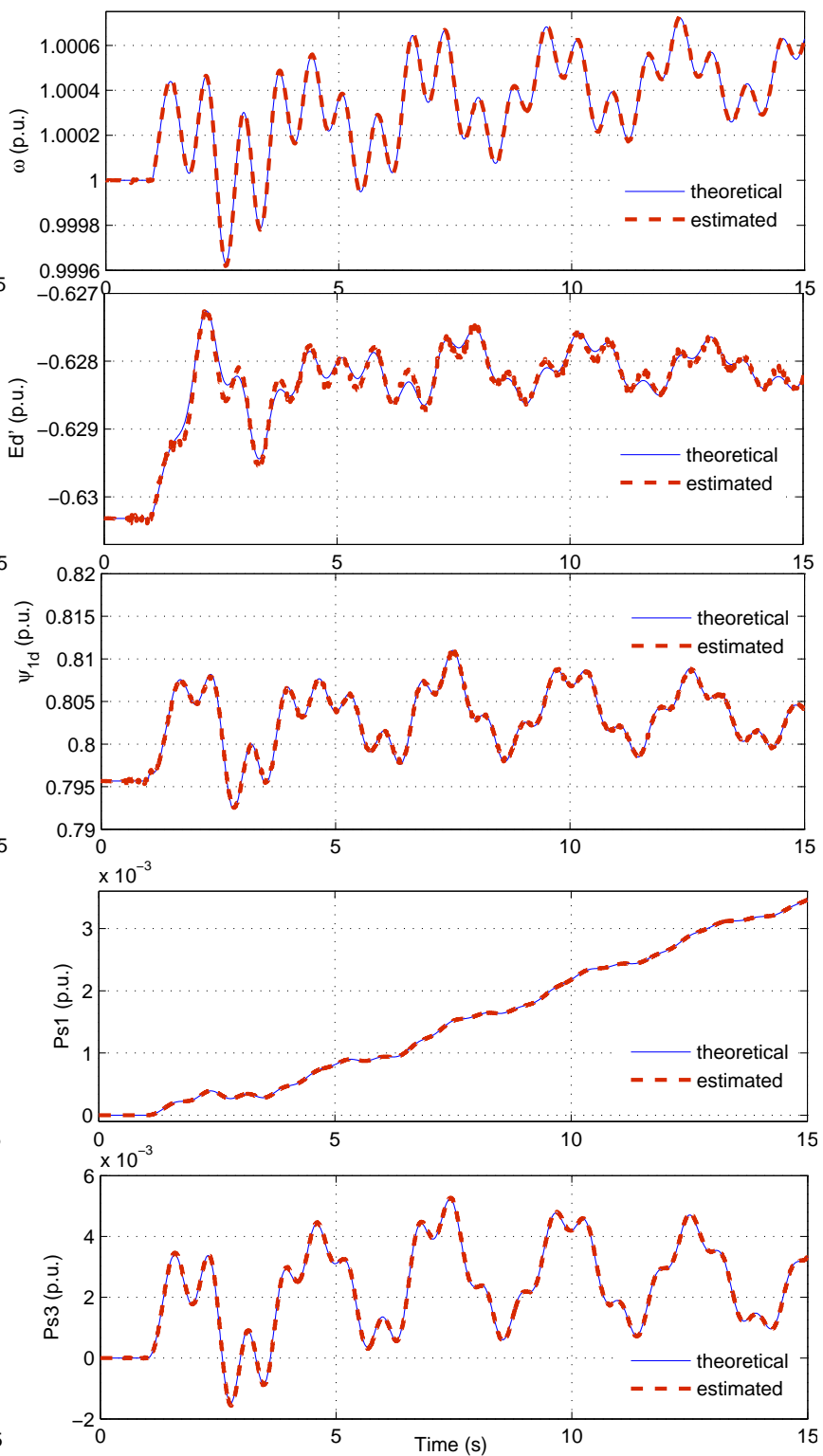

Fig. 5. Estimated vs theoretical values for dynamic states of the ninth generation unit 
diagonal element of $\boldsymbol{P}_{y i}^{-}$; and $P_{y i, 2}^{-}$is the second diagonal element of $\boldsymbol{P}_{y i}^{-}$.

$$
\begin{aligned}
& \left|\lambda_{y_{i, 1}}\right|<\lambda_{0} ; \text { where } \lambda_{y_{i, 1}}=\frac{y_{i, 1}-\hat{y}_{i, 1}^{-}}{\sqrt{P_{y i, 1}^{-}}} \\
& \left|\lambda_{y_{i, 2}}\right|<\lambda_{0} ; \text { where } \lambda_{y_{i, 2}}=\frac{y_{i, 2}-\hat{y}_{i, 2}^{-}}{\sqrt{P_{y i, 2}^{-}}}
\end{aligned}
$$

$\lambda_{0}$ depends on the type of system, and it may be found using off-line simulations [11], [12]. For the system in case study, $\lambda_{0}$ was found to be 10 . Hence, a measurement is labeled as a bad measurement if its normalized innovation ratio comes out to be more than $\lambda_{0}$ in a given sample, and is thus discarded and the actual measurement is assumed to be same as the predicted measurement for that sample.
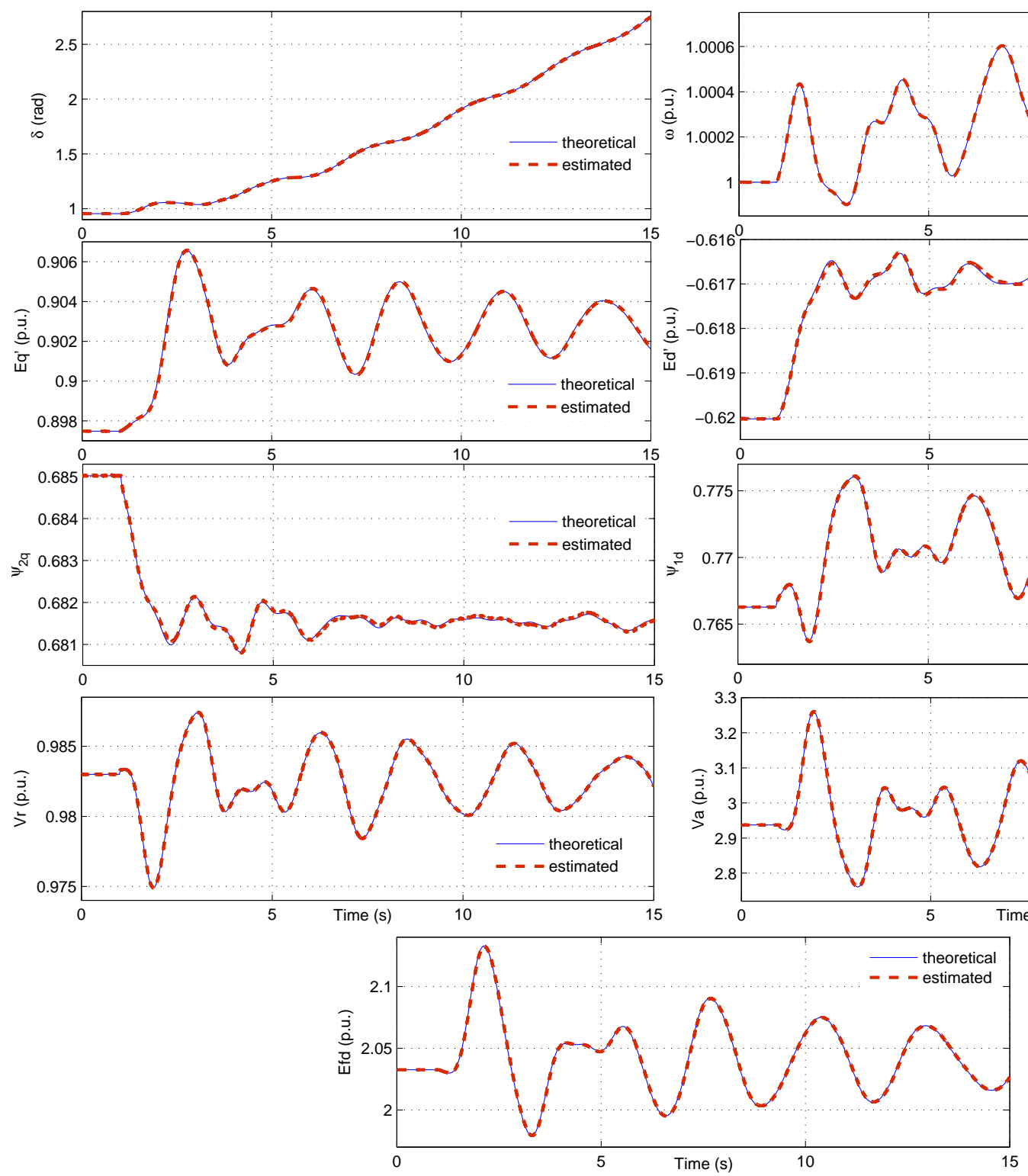

Fig. 6. Estimated vs theoretical values for dynamic states of the third generation unit
The above technique for bad data detection would have worked flawlessly if there wasn't any bad data present in the states or input. But since pseudo-inputs are used in the decentralized UKF algorithm, which are in reality measurements, bad-data may also be present in these pseudo-inputs. Innovation ratios are not defined for pseudo-inputs, and hence we cannot directly detect bad-data in them; but an indirect method may be used to do so. This method is based on the fact that the predicted measurements are influenced by bad-data in the pseudo-inputs but the actual measurements remain independent of these bad-data, and hence in the case of bad-data in pseudo-inputs no correlation exists between the actual measurements and the predicted measurements. In other words, if bad data is introduced in one or more pseudo-input(s) in a given sample, then both $\hat{\boldsymbol{y}}_{i}^{-}$and $\boldsymbol{P}_{y i}^{-}$would change significantly from their correct values, and this change will be completely uncorrelated with $\boldsymbol{y}_{i}$, even if bad data is present
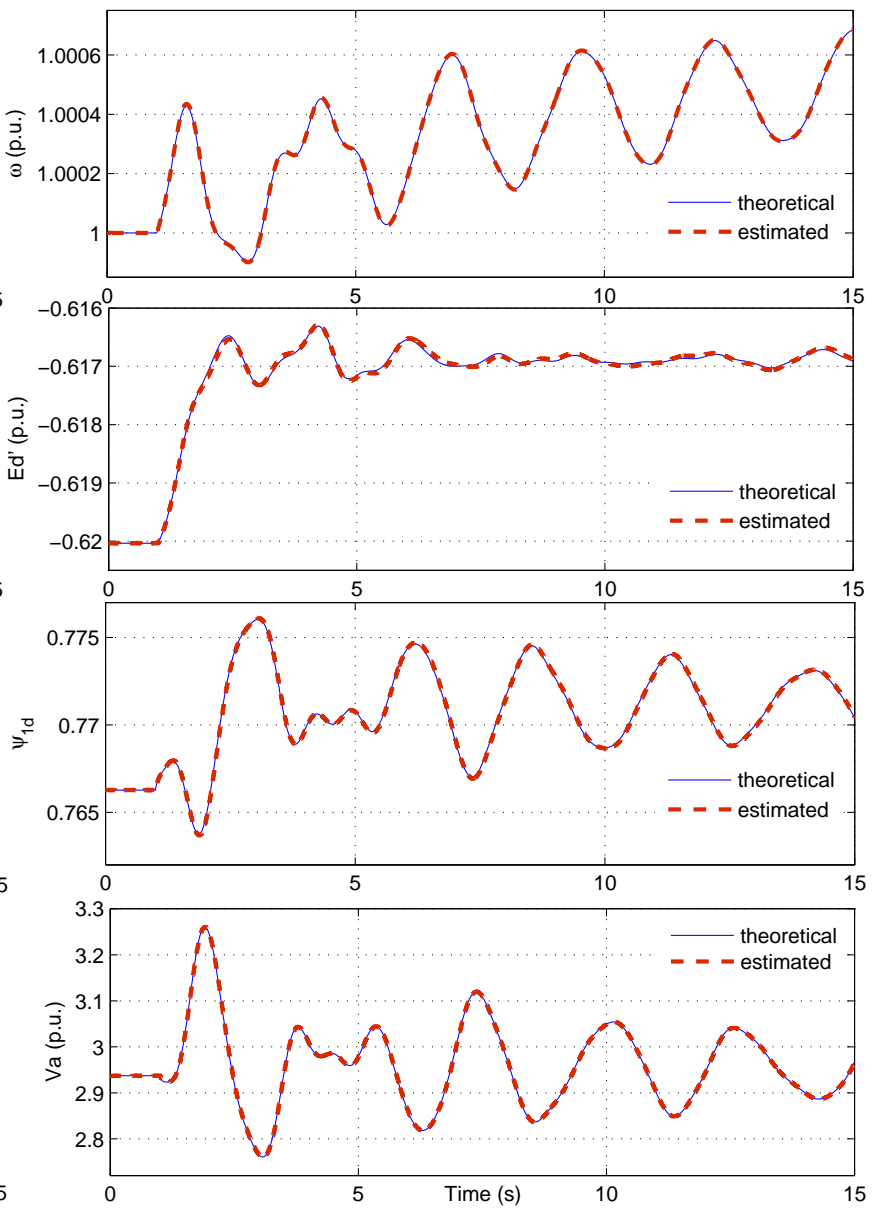
in $\boldsymbol{y}_{i}$ as well (assuming that all the bad-data are introduced randomly and independently), and thus the values of both $\lambda_{y_{i, 1}}$ and $\lambda_{y_{i, 2}}$ are expected to exceed $\lambda_{0}$ in such an event. Thus, we need to modify the technique in the previous paragraph, and discard all the pseudo-inputs if both $\lambda_{y_{i, 1}}$ and $\lambda_{y_{i, 2}}$ exceed $\lambda_{0}$ in a given sample, and use the latest uncorrupted pseudoinputs instead. Thus, the bad-data detection for the $k^{t h}$ sample takes place according to the following algorithm:

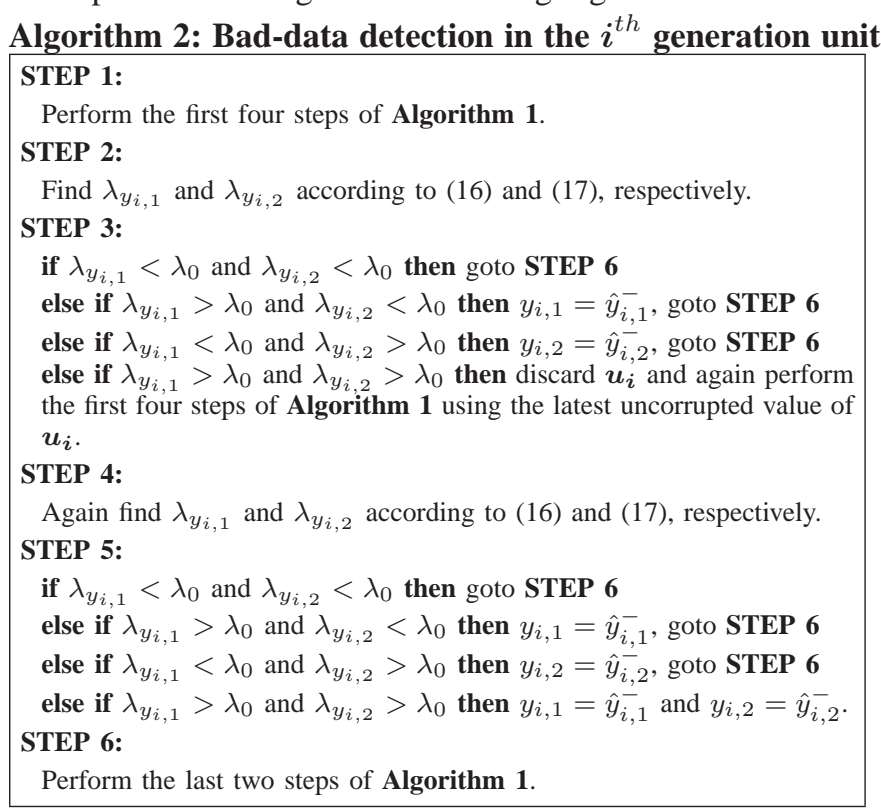

A bad-data detector based on Algorithm 2 was implemented and integrated in the decentralized UKF algorithm. The values of $\lambda_{y_{i, 1}}$ and $\lambda_{y_{i, 2}}$, and the estimated rotor velocity for $i=13$, have been shown for three cases, all in Fig. 8
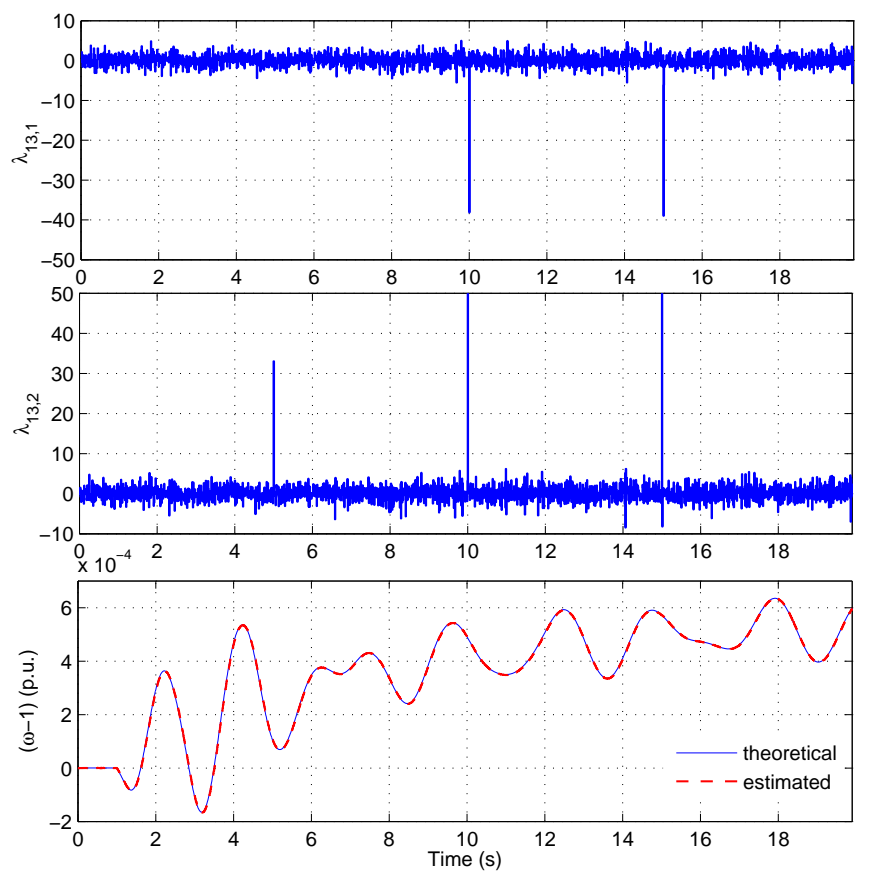

Fig. 8. Bad-data detection

- Bad-data present only in one of the measurements: In this case bad-data is introduced in the measurement $\phi_{y 13}$ of magnitude +0.01 p.u. (i.e. the measured value of $\phi_{y 13}$ is 0.01 p.u. above its true value), at time $t=5 \mathrm{~s}$. It may be observed that the bad-data detector effectively handles this anomaly, and there is no effect on $\lambda_{y_{13,1}}$, and the estimation process remains unaffected.

- Bad-data present only in one of the pseudo-inputs: In this case bad-data is introduced in the pseudo-input $V_{y 13}$ of magnitude -0.01 , at time $t=10 \mathrm{~s}$. It may be observed that both $\lambda_{y_{13,1}}$ and $\lambda_{y_{13,2}}$ become unbounded, but the bad-data detector effectively handles this anomaly as well, as the estimation process remains unaffected.

- Bad-data present simultaneously in one of the measurements and in one of the pseudo-inputs: In this case baddata is introduced in the measurement $\phi_{y 13}$ of magnitude +0.01 p.u., and another bad-data is introduced in the pseudo-input $V_{y 13}$ of magnitude -0.01 p.u., both at $t=15 \mathrm{~s}$. It may be observed that both $\lambda_{y_{13,1}}$ and $\lambda_{y_{13,2}}$ become unbounded, as in previous case, but the bad-data detector effectively handles this anomaly as well.

\section{CONCLUSIONS}

A scheme for decentralized estimation of the dynamic states of a power system has been proposed. The scheme preserves the non-linearity in the system and improves efficiency over other non-linear filters through unscented Kalman filtering. The basic idea of decentralization in the scheme is based on treating some of the measured signals as pseudo inputs. The advantages of the proposed scheme over the centralized schemes have been presented in terms of speed, feasibility, simplicity and high accuracy. The scheme is also robust to moderately-high noise levels and gross errors in the measurement signals. It is the belief of the authors that the proposed scheme will serve as a highly practical method of dynamic state estimation for dynamic security assessment in modern power systems.

\section{APPENDIX A}

DISCRETE DAES FOR THE POWER SYSTEM MODEL

The discrete DAEs for the sub-transient model of a power system are given by (18)-(24).

\section{REFERENCES}

[1] Y. Xu, Z. Y. Dong, J. H. Zhao, P. Zhang, K. P. Wong, "A Reliable Intelligent System for Real-Time Dynamic Security Assessment of Power Systems," IEEE Trans. Power Syst., vol. 27, no. 3, pp. 12531263, Aug. 2012.

[2] D. Z. Fang, A. K. David, C. Kai, C. Yunli, "Improved hybrid approach to transient stability assessment," IEE Proceedings-Gener. Transm. and Dist., vol. 152, no. 2, pp. 201-207, Mar. 2005.

[3] N. Fernandopulle, R. T. H. Alden, "Improved dynamic security assessment for AC/DC power systems using energy functions," IEEE Trans. Power Syst., vol. 18, no. 4, pp. 1470-1477, Nov. 2003.

[4] M. A. El-Kady, C. K. Tang, V. F. Carvalho, A. A. Fouad, V. Vittal, "Dynamic Security Assessment Utilizing the Transient Energy Function Method," IEEE Trans. Power Syst., vol. 1, no. 3, pp. 284-291, 1986.

[5] E. Ghahremani, I. Kamwa, "Dynamic State Estimation in Power System by Applying the Extended Kalman Filter With Unknown Inputs to Phasor Measurements," IEEE Trans. Power Syst., vol. 26, no. 4, pp.25562566, 2011.

[6] G. K. Gharban, B. J. Cory, "Non-linear dynamic power system state estimation," IEEE Trans. Power Syst., vol. 1, no. 3, pp. 276-283, 1986. 
[7] W. Miller, J. Lewis, "Dynamic state estimation in power systems," IEEE Trans. Autom. Control, vol. 16, no. 6, pp. 841-846, Dec. 1971.

[8] Z. Huang, K. Schneider, J. Nieplocha, "Feasibility studies of applying Kalman Filter techniques to power system dynamic state estimation," IPEC 2007. Int. Power Eng. Conf., pp.376-382, 3-6 Dec. 2007.

[9] P. Du, Z. Huang, R. Diao, B. Lee, K. K. Anderson, "PMU Placement for enhancing dynamic observability of a power grid," Innovative Technologies for an Efficient and Reliable Electricity Supply (CITRES), 2010 IEEE Conference on, pp.15-21, 27-29 Sept. 2010.

[10] E. Ghahremani, I. Kamwa, "Online State Estimation of a Synchronous Generator Using Unscented Kalman Filter From Phasor Measurements Units," IEEE Trans. Energy Conv., vol. 26, no. 4, pp.1099-1108, 2011.

[11] S. Wang, W. Gao, A.P.S. Meliopoulos, "An Alternative Method for Power System Dynamic State Estimation Based on Unscented Transform," IEEE Trans. Power Syst., vol. 27, no. 2, pp. 942-950, May 2012.

[12] G. Valverde, V. Terzija, "Unscented kalman filter for power system dynamic state estimation," IET Gener., Transm. and Dist. vol. 5, no. 1, pp.29-37, Jan. 2011.

[13] A. G. Phadke, "Synchronized phasor measurements in power systems," IEEE Computer Applications in Power, vol.6, no.2, pp.10-15, Apr. 1993.

[14] IEEE Standard for Synchrophasor Measurements for Power Systems, IEEE Std C37.118.1-2011, Dec. 2011.

[15] J. K. Uhlmann, "Simultaneous map building and localization for realtime applications," Transfer thesis. University of Oxford, 1994.

[16] S. J. Julier, J. K. Uhlmann, "A new extension of the Kalman filter to nonlinear systems," The Proceedings of AeroSense: The 11th International Symposium on Aerospace/Defense Sensing, Simulation and Controls, Orlando, Florida, SPIE, 1997.

[17] R. E. Kalman, "A New Approach to Linear Filtering and Prediction Problems," Journal of Basic Engineering, vol. 82 D, pp. 35-45, 1960.

[18] S. Julier, J. Uhlmann, H. F. Durrant-Whyte, "A new method for the nonlinear transformation of means and covariances in filters and estimators," IEEE Trans. Autom. Control, vol. 45, no. 3, pp. 477-482, Mar. 2000.

[19] B. Pal and B. Chaudhuri, Robust Control in Power Systems. New York, U.S.A.: Springer, 2005, chapter 4.

[20] M. A. Pai and P. W. Sauer, Power System Dynamics and Stability. New Jersey, U.S.A.: Prentice Hall, 1998, chapter 6.

[21] I. Markovsky and B. D. Moor, "Linear dynamic filtering with noisy input and output," Automatica, vol. 41, pp. 167-171, 2005.

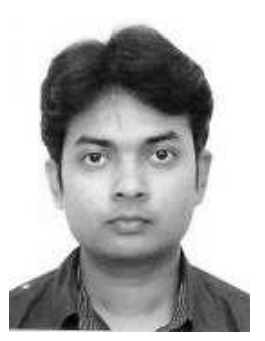

Abhinav Kumar Singh (S'12) received the B.Tech. degree from the Indian Institute of Technology, New Delhi, India, in 2010 in electrical engineering (power).

From 2010-2011 he worked at the Indian Institute of Technology, New Delhi, India, as a Research Assistant. Currently, he is pursing the Ph.D. degree at Imperial College London, London, U.K. His current research interests include state estimation, networked control and communication aspects of power systems.

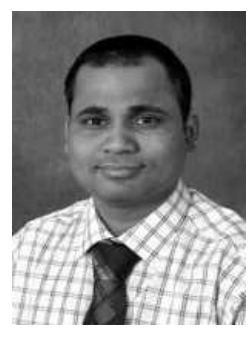

Bikash C. Pal (M'00-SM'02-F'13) received the B.E.E. (with honors) degree from Jadavpur University, Calcutta, India, the M.E. degree from the Indian Institute of Science, Bangalore, India, and the Ph.D. degree from Imperial College London, London, U.K., in 1990, 1992, and 1999, respectively, all in electrical engineering.

Currently, he is a Professor in the Department of Electrical and Electronic Engineering, Imperial College London. He is Editor-in-Chief of IEEE Transactions on Sustainable Energy and Fellow of IEEE for his contribution to power system stability and control. His current research interests include state estimation, and power system dynamics. 